\title{
Spiral arrangement: From nanostructures to packaging
}

\author{
Vladimír Kolařík, Miroslav Horáček, Alexandr Knápek, \\ Stanislav Krátký, Milan Matějka, Petr Meluzín*
}

\begin{abstract}
Although the orthogonal systems are predominantly employed within modern technologies, some general systems can be found principally in nature. Progress in the field of nanotechnologies based on a condensed matter will reach its limit at a certain moment, which may be caused by material limits or limits of manufacturing technologies. This limitation will affect both approaches, the top-down as well as the bottom-up. Another way to obtain a nanostructure will be based purely on nature and its ability to grow, which requires a deep understanding of the world of biology. This natural approach is closely connected with a precise mathematical description which is necessary for employment of both the analytical and synthetic tools which are presently available within the frame of current technological methods. In this paper, we present an analysis of a model based on a spiral arrangement on a series of elements.
\end{abstract}

K e yw ords: electron beam lithography, phyllotaxis, spiral arrangement, parastichy

\section{Introduction}

The arrangement comes originally from biology where the placement of seeds in the pseudanthium is studied within the field called Phyllotaxy. As a result, the phyllotactic model describes the positions of seeds in a flower head, which are, in our case, the positions of optical elements created by the e-beam lithography on the substrate. The seed positions in polar coordinates can be described by an equation presented by Vogel [1]

$$
\left\{r_{k} ; \varphi_{k}\right\}=\left\{c k^{0.5} ; k \varphi_{0}\right\}
$$

where $k$ is the rank of a seed in the sequence, $c$ is the scale factor and $\varphi_{0}$ is the angular factor.

Among other noticeable features, there are two sets of secondary spirals, also known as parastichies, one turning clockwise, the other counterclock-wise, which are composed of the nearest neighboring seeds. Although there

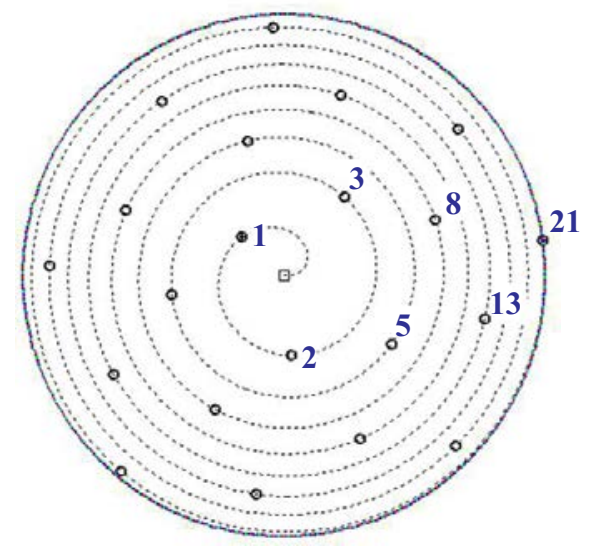

are still many aspects that should be further justified, Vogels equation correctly describes the arrangement of seeds visible in an actual flower head, Fig. 1(a). For this reason, this model attracts attention not only to biologists and mathematicians but also has been used for various purposes in the past decade, eg for the mirror distribution pattern for Gemasolar solar plant located in Spain or for benchmarking pattern for e-beam lithography [2-4]. When the structure is created within the nanometer scale, the situation becomes even more interesting as there are many diffraction-related effects [5] taking place and the structure shows an interesting behavior from the point of view of reflection, refraction, and absorption, Fig. 1(b), [6]. The topic has already been solved from the point of view of photonics, discussing mainly the scattering properties of the three main types of deterministic aperiodic spiral arrays of $\mathrm{Au}$ nanoparticles and also studying the

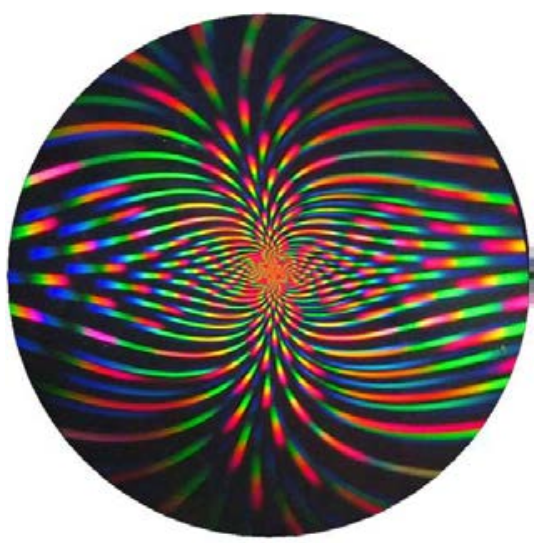

Fig. 1. (a) - Vogels model, and (b) - diffractive pattern of the planar optical phyllotactic arrangement showing higher-order diffraction, a picture of the real sample

* Institute of Scientific Instruments of the CAS, v. v. i., Královopolská 147, 61264 Brno, Czech Republic, vladimir.kolarik@isibrno.cz 

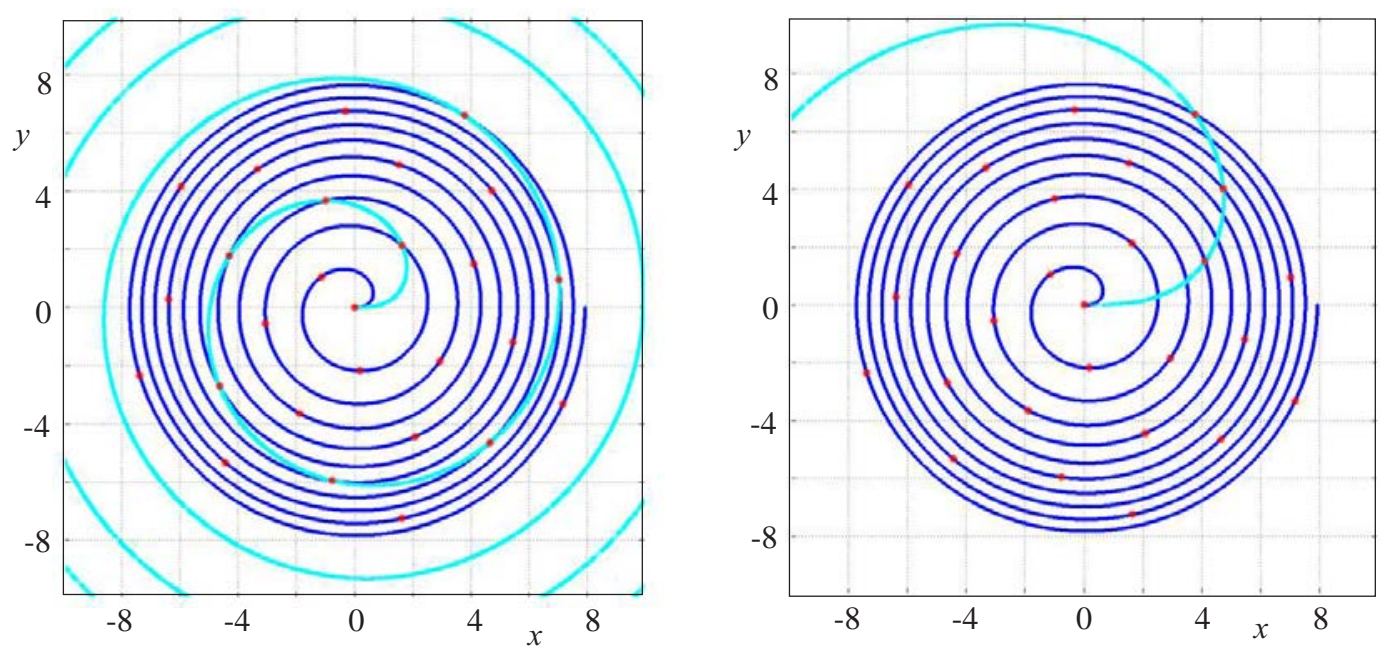

Fig. 2. (left)- illustration of the secondary spiral for $F_{\mathrm{n}}=3$, (right) - illustration of the secondary spiral for $F_{n}=8$

Table 1. Numerical results achieved by equations 7-12

\begin{tabular}{lccccc}
\hline & \multicolumn{2}{c}{$F_{n}$} & \multicolumn{2}{c}{$a_{F_{n}}$} & \multicolumn{2}{c}{$1 / a_{F^{2}}$} \\
Even & Odd & Even & Odd & Even & Odd \\
\hline 3 & - & 2.8025 & - & 0.1273 & - \\
- & 5 & - & 4.6022 & - & -0.0472 \\
8 & - & 7.4049 & - & 0.0182 & - \\
- & 13 & - & 12.0072 & - & $-0,0069$ \\
21 & - & 19.4121 & - & 0.0027 & - \\
- & 34 & - & 34.4193 & - & -0.0010 \\
\hline
\end{tabular}

circular symmetry in continuous Fourier space of Vogel's spirals [7].

\section{Secondary spirals}

Considering a trajectory of the primary spiral described by (1), where

$$
\begin{gathered}
\varphi=t \\
r=a \sqrt{t}
\end{gathered}
$$

Consequently, a sampling of the spiral $t_{0}$ is taken into account and expressed as

$$
t_{0}=\frac{2 \pi}{\Phi^{2}}, \quad \Phi=\frac{1+\sqrt{5}}{2}
$$

and where the samples coordinates are expressed by

$$
\begin{gathered}
\varphi_{k}=k t_{0} \\
r_{k}=a \sqrt{k t_{0}}
\end{gathered}
$$

We are looking for a function whose trajectory goes through a selected subset of the samples. Let us consider each $F_{n}$-th point, where $F_{n}$ can get a value of an even term of a progression $\{1,1,2,3,5,8,13,21,34, \ldots\}$ starting with the 4 -th term, thus $F_{n}$ is $\{3,8,21,55,144, \ldots\}$ . The desired function can be viewed from two perspectives: either the trajectory unwinds more slowly in angular terms, where

$$
\begin{aligned}
\varphi_{F_{n}} & =\frac{1}{a_{F_{n}}^{2}} t \\
r_{F_{n}} & =a \sqrt{t}
\end{aligned}
$$

or the radius grows faster, where

$$
\begin{gathered}
\varphi_{F_{n}}=t \\
r_{F_{n}}=a_{F_{n}} a \sqrt{t}
\end{gathered}
$$

Let us consider the first mentioned expression (7) and (8). Considering a sample Fn which is located at the angle $\varphi_{k=F_{n}}=F_{n} t_{0}$ of the primary spiral. For the secondary spiral, this angle is decreased by a whole-number multiple of $2 \pi$. It can be demonstrated that this integer multiple of $F_{n-2}$ equals to: $\varphi_{F_{n}}=\varphi_{k=F_{n}}-2 \pi F_{n-2}$. The ratio of these two angles determines the velocity of rotation expressed by a coefficient in (7)

$$
\begin{aligned}
& \frac{\varphi_{k=F_{n}}}{\varphi_{F_{n}}}=\frac{F_{n} t_{0}}{F_{n} t_{0}-2 \pi F_{n-2}}= \\
& =\frac{1}{1-\frac{2 \pi F_{n-2}}{F_{n} t_{0}}}=\frac{1}{1-\frac{2 \pi F_{n-2}}{F_{n} \frac{2 \pi}{\Phi^{2}}}}= \\
& =\frac{1}{1-\frac{F_{n-2}}{F_{n}} \Phi^{2}}=a_{F_{n}}^{2}
\end{aligned}
$$

A ratio of the velocity increase (described by a coefficient in (10) is expressed by

$$
a_{F_{n}}=\frac{1}{\sqrt{1-\frac{F_{n-2}}{F_{n}} \Phi^{2}}}
$$



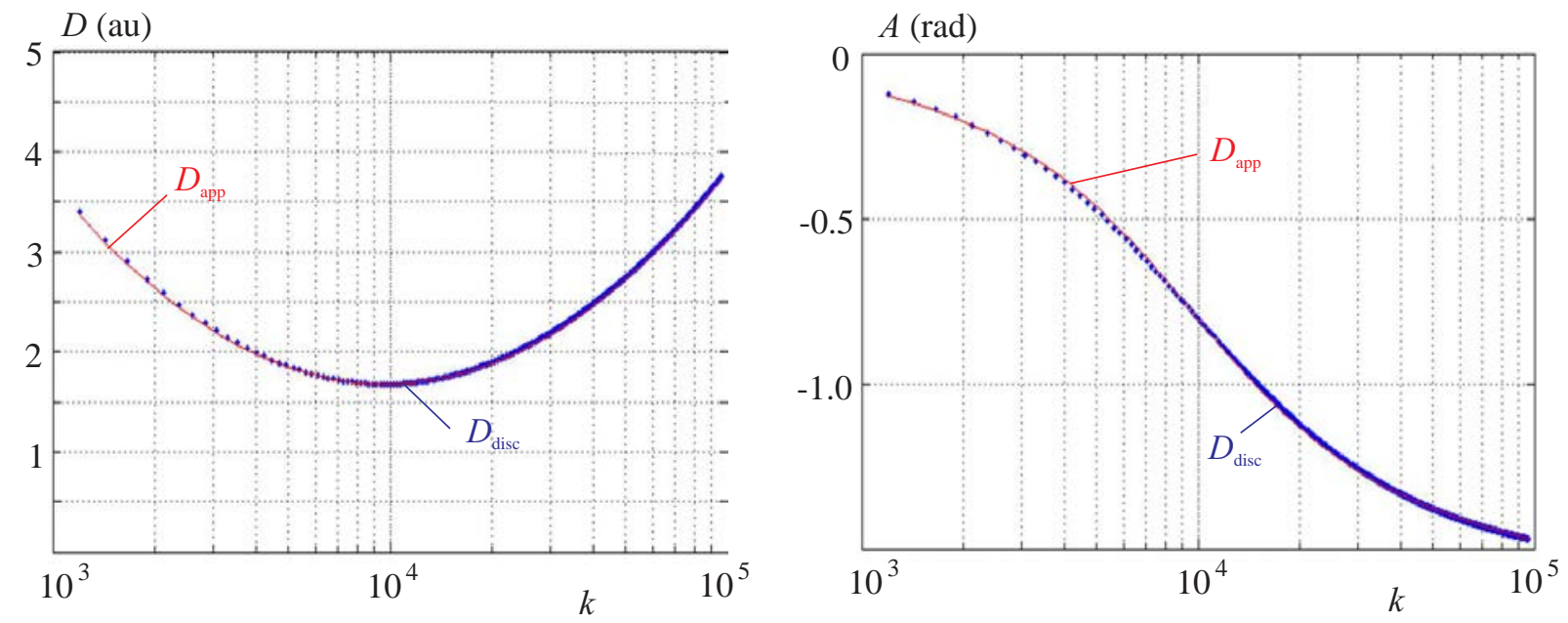

Fig. 3. Distance of adjacent seeds and inclination of the secondary spiral, $n=13, F_{n}=233$

For the even terms of Fibonacci progression (starting with the 3 rd term) $\{2,5,13,34,89, \ldots$,$\} , the rotation di-$ rection is opposite: $\varphi_{F_{n}}=-\frac{1}{a_{F_{n}}^{2}} t$ according to $(7)$ or more precisely $\varphi_{F_{n}}=-t$ according to (9). Correspondingly, the absolute value should be considered for the (7), yielding

$$
a_{F_{n}}=\frac{1}{\sqrt{\left|1-\frac{F_{n-2}}{F_{n}} \Phi^{2}\right|}} .
$$

The numerical results are illustrated in the following Tab. 1 and in Fig. 2 for $F_{\mathrm{n}}=3$ and $F_{\mathrm{n}}=8$.

\section{Analysis of the interelement positions}

Discrete calculation based on a $x, y$ coordinates of the primary spiral elements which are located between $k$-th element and an adjacent $\left(k+F_{\mathrm{n}}\right)$-th element along the secondary spiral (sampled $F_{n}$ )

$$
D_{d i s}=\sqrt{\left(x_{k+F_{n}}-x_{k}\right)^{2}+\left(y_{k+F_{n}}-y_{k}\right)^{2}}
$$

An approximated continuous function $D_{a p p}=c_{2} \xi^{2}+$ $c_{1}$, with a substitution of $\xi=\log \left(k / k_{\operatorname{char} F_{n}}\right)^{2}$, where $k$ stands for an element number within the sampled primary spiral, $k_{\mathrm{char} F_{n}}$ is a characteristic element of the secondary spiral (the minimal distance between neighboring elements along the secondary spiral and also a slope of the secondary spiral towards the position vector, approximately 45 degrees)

$$
k_{\mathrm{char} F_{n}}=F_{n}^{2} \frac{f_{m}}{2 \pi}
$$

and with coefficients

$$
\begin{aligned}
& c_{1}=\sqrt{\frac{\pi}{f_{m}}}, \quad f_{m}=\frac{\Phi+\Phi^{-1}}{2} \\
& \Phi=1+\frac{\sqrt{5}}{2}, \quad c_{2}=\frac{\Phi}{\pi}
\end{aligned}
$$

\subsection{Slope of the secondary spiral towards the position vector}

Discrete slope of the connecting line between the $k$-th element and the $(k+F n)$-th element (thus towards the position vector of the spiral in a particular element (thus towards the angular coordinate of the $k$-th element)

$$
A_{\mathrm{dis}}=\arctan \frac{y_{k+F_{n}}-y_{k}}{x_{k+F_{n}}-x_{k}}-\varphi_{k}
$$
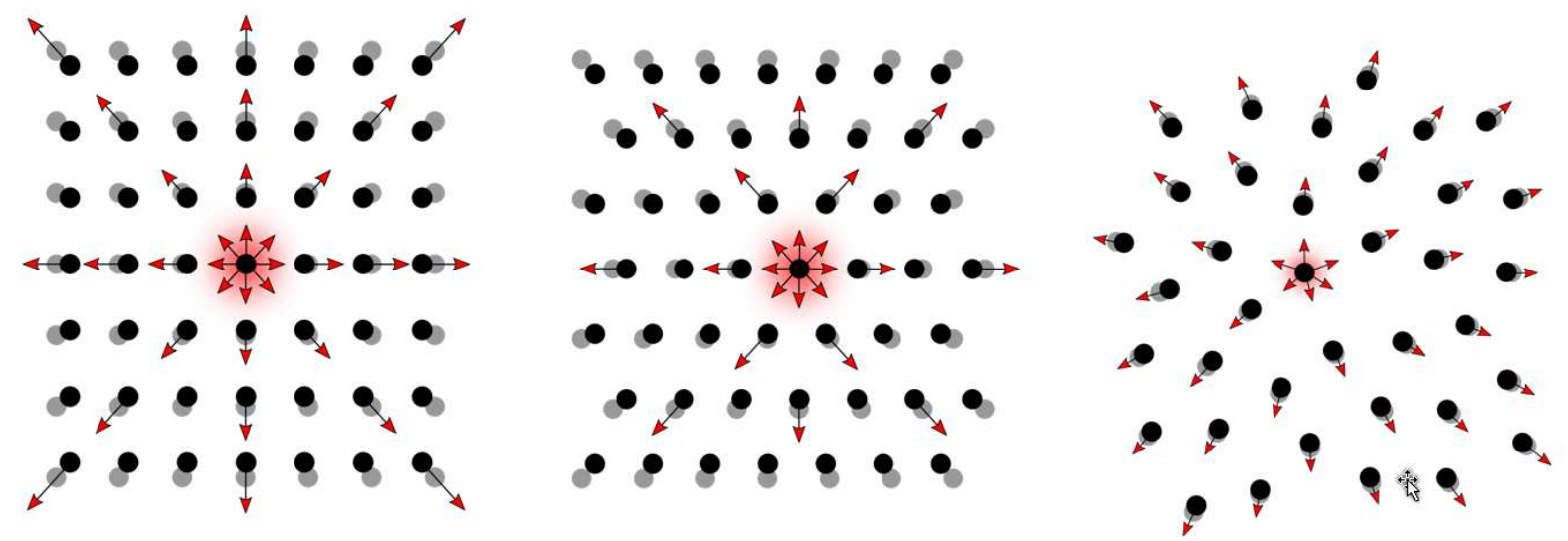

Fig. 4. A simplified analysis of the heat distribution within: (a) - the common orthogonally ordered package,(b) - hexagonally ordered package, and (c) - non-orthogonal arrangement based on the phyllotactic model 
Approximation continuous function

$$
\begin{aligned}
& A_{\text {app }}=c_{3} \arctan \xi+c_{4} \\
& \text { with coefficients: } c_{3}=\frac{1}{\Phi}, c_{4}=\frac{\pi}{4}
\end{aligned}
$$

the substitution remains the same for (13). Numerically, the coefficients equal: $c_{3}=0.6180, c_{4}=0.7854$. Situation is illustrated in Fig. 3.

\section{Phyllotaxy pin distribution of packages}

A possibility of pin distribution of packages that are intended for integrated circuits within the microelectronics field and contain an extensive number of pins, based on the phyllotactic model is presented in Fig. 4. Based on the simplified analysis, the phyllotactic arrangement of pins, Fig. 4(b), may provide lower stress induced due to thermal expansions in comparison to a common orthogonal system of pin placement, Fig. 4(a). The presented mathematical description of the secondary spirals allows to compare the numerical model and the exact analytical description of the thermal flow in package.

\section{Conclusion}

The paper deals with the mathematical description of some aspects of the phyllotaxy arrangement of elements based on the Vogels model of phyllotactic structures. The secondary spirals were described, the interelement positions along the spirals and slope of the secondary spirals were analyzed. The rigorous mathematical analysis and description of the phyllotaxy allows for a future use of this natural model in a wide spectra of devices from the macro world to nanotechnologies.

\section{Acknowledgements}

The research was supported by the Ministry of Industry and Trade (project no. FV10618), by the Technology Agency of the Czech Republic (projects no. TG03010046 and TE01020233) and by the Ministry of Education, Youth and Sports of the Czech Republic (project no. LO1212). The research infrastructure was funded by the Ministry of Education, Youth and Sports of the Czech Republic and the European Commission (project CZ.1.05/2.1.00/01.0017), and by the Czech Academy of Sciences (project RVO:68081731).

\section{REFERENCES}

[1] H. Vogel, Mathematical biosciences, 44.3-4, pp. 179-189, 1979,.

[2] M. Horacek, P. Meluzin, S. Kratky, M. Matejka and V. Kolarik, "Phyllotactic Arrangements of Optical Elements", Proceedings of SPIE 10233, pp. 1-7, 2017.

[3] S. Kratky, M. Urbanek and V. Kolarik, "PEC Reliability in 3D E-beam DOE Nanopatterning", Microscopy and Microanalysis 21, pp. 230-235, 2015.
[4] P. Meluzin, M. Horacek, M. Urbanek, J. Bok, S. Kratky, M. Matejka, J. Chlumska and V. Kolarik, "Some Other Gratings: Benchmarks for Large-Area E-Beam Nanopatterning, Proceedings of the 6th International conference NANOCON 2014 pp. 246-251, 2014.

[5] V. Kolarik, M. Horacek, M. Urbanek, M. Matejka, S. Kratky, J. Chlumska and J. Bok, "Structural Color of Metallic Surfaces", Proceedings of the 23rd International conference METAL 2014, pp. 962-967, 2014.

[6] R. L. Van Renesse, Optical document security 3rd edition, 1st ed. Boston: Artech House, pp. 177-222, 2005.

[7] J. Trevino, H. Cao and L. Dal Negro, "Circularly Symmetric Light Scattering from Nanoplasmonic Spirals", Nano Letters 11, pp. 2008-2016, 2011.

Received 26 November 2018

Vladimír Kolařík (Assoc Prof) was born in 1966 in Brno, Czech Republic. He received his $\mathrm{PhD}$ in microelectronics from the Institute National Polytechnique de Grenoble (France) in 1994. Currently, he works as a researcher at the Institute of Scientific Instruments of the Czech Academy of Sciences. He is internationally known for his work in the field of electron beam lithography, especially within the field of designing function nanostructures and planar optical devices.

Miroslav Horáček (PhD) was born in 1964 in Brno, Czech Republic. He received his PhD in electronics technology from Brno University of Technology in 1999 with the research involving the low energy electron microscopy. Currently, he works as a researcher at the Institute of Scientific Instruments of the Czech Academy of Sciences where he deals mainly with the electron beam lithography and electron emitters.

Alexandr Knápek (PhD) was born in 1983 in Brno, Czech Republic. He received his PhD from Brno University of Technology in Brno in 2013. Currently, he works as a researcher at the Institute of Scientific Instruments of the Czech Academy of Sciences where he deals mainly with the experimental research of the new cold field emission emitters. His research interest also includes microscopy inspection techniques, instrumentation, and non-orthogonal planar systems.

Stanislav Krátký (PhD student) was born in 1987 in Ivancice, Czech Republic. He is currently studying (PhD) at Brno University of Technology in Brno. He works as a postgraduate student at the Institute of Scientific Instruments of the Czech Academy of Science where he mainly deals with electron beam lithography. His research is also focused on diffractive optics and other micro and nanotechnologies such as reactive ion etching, UV lithography and various techniques for thin layers preparation.

Milan Matějka (PhD) was born in 1982 in Brno, Czech Republic. He received his PhD from Brno University of Technology in Brno in 2016. Currently, he works as a post-doctoral researcher at the Institute of Scientific Instruments of the Czech Academy of Sciences where he deals with the experimental and application research in the field of electron beam lithography (EBL), Micro- and Nano- fabrication technologies. In his research activities, he also deals with the development of micro electromechanical systems (MEMS) and optically variable devices (OVDs).

Petr Meluzín was born in 1988 in Boskovice, Czech Republic. He studied and finished his masters degree at Mendel university in Brno. Currently, he works as a programmer at the Institute of Scientific Instruments of the Czech Academy of Sciences where he deals mainly with the data preparation and software programming for electron-beam lithography. 